\title{
Risk factors for hemoglobinuria after ultrasonography-guided percutaneous microwave ablation for large hepatic cavernous hemangiomas
}

\author{
Fangyi Liu ${ }^{1}$, Xiaoling Yu ${ }^{1}$, Zhigang Cheng ${ }^{1}$, Zhiyu Han ${ }^{1}$, Jianping Dou ${ }^{1}$, Jie $\mathrm{Yu}^{1}$ and \\ Ping Liang ${ }^{1}$ \\ ${ }^{1}$ Department of Interventional Ultrasound, Chinese PLA General Hospital, Beijing, 100853 China \\ Correspondence to: Ping Liang, email: liangping301@hotmail.com
}

Keywords: microwave ablation; hepatic cavernous hemangioma; ultrasound guidance; hemoglobinuria; risk factors Received: September 07, 2017 Accepted: October 29, $2017 \quad$ Published: May 22, 2018

Copyright: Liu et al. This is an open-access article distributed under the terms of the Creative Commons Attribution License 3.0 (CC BY 3.0), which permits unrestricted use, distribution, and reproduction in any medium, provided the original author and source are credited.

\section{ABSTRACT}

Thermal ablation of large hepatic cavernous hemangiomas may lead to intravascular hemolysis, hemoglobinuria, and even acute renal failure. This study aimed to identify the risk factors associated with hemoglobinuria after ultrasonography-guided percutaneous microwave ablation for large hepatic cavernous hemangiomas. In our study, 11 related risk factors were analyzed using univariate and multivariate binary logistic regression model and Receiver operating characteristic curves to determine the contribution to hemoglobinuria after microwave ablation for 49 patients with 51 hepatic cavernous hemangiomas. By multivariate analysis, the ablation time ( $p=0.021$; Odds Ratio, 1.005$)$, and the number of antenna insertions $(p=0.036$; Odds Ratio, 3.568$)$ were the independent risk factors associated with hemoglobinuria. The cutoff value for ablation time and the number of antenna insertions in predicting the presence of hemoglobinuria was 1185 s (sensitivity, 75\%; specificity, 69\%) and 4.5 (sensitivity, 55\%; specificity, 83\%), respectively. Less than 5 of antenna insertions and less than $\mathbf{2 0}$ mins of ablation time may therefore be recommended in patients with microwave ablation of large hepatic cavernous hemangiomas, in order to reduce the occurrence of hemoglobinuria. This is the first report about the risk factors analysis associated with hemoglobinuria after thermal ablation for large hepatic cavernous hemangiomas.

\section{INTRODUCTION}

Hepatic cavernous hemangiomas (HCHs) are the most common benign neoplasms in liver, with the prevalence in the general population as many as $20 \%$ in autopsy studies [1]. Hemangiomas are referred to as "giant" if greater than $4 \mathrm{~cm}$ in diameter [2]. Although most hemangiomas are asymptomatic and can be managed safely with observation alone, larger lesions may produce a variety of symptoms and signs, including abdominal pain, dyspepsia, jaundice, thrombocytopenia and even spontaneous rupture $[3,4]$. The primary treatment is surgical resection, transarterial embolization, radiation therapy, and the use of a vascular endothelial growth factor (VEGF) inhibitor have also been reported [5-8]. Thermal ablation, such as radiofrequency ablation (RFA) and microwave ablation (MWA), was safe, well-tolerated, and effective in markedly shrinking large $\mathrm{HCHs}$ and improving symptoms in most patients [9-12]. Hemoglobinuria after thermal ablation of HCHs was a common side effect and and should be taken seriously. Hemoglobinuria is a type of abnormal urine (deep-yellow-colored or winecolored) with hemoglobin ( $\mathrm{Hb})$, which is often found in intravascular hemolysis leading to cell-free $\mathrm{Hb}$ released into circulatory system [13]. Because hemangiomas are sinusoids and the main component inside is the blood, thermal ablation by inserting the ablation needle into the tumor can lead to massive red cell destruction, intravascular hemolysis, and even acute kidney injury. Van Tilborg AA et al. reported two patients with very large symptomatic $\mathrm{HCHs}$ who developed acute kidney injury (AKI) shortly after bipolar RF ablation, caused by massive heat-induced 
intravascular hemolysis [14]. Determining risk factors associated with hemoglobinuria after thermal ablation for large hepatic cavernous hemangiomas is beneficial for reducing the risk of ablation. To our knowledge, there has been no report about the risk factors analysis associated with hemoglobinuria after thermal ablation for large hepatic cavernous hemangiomas. This study aimed to identify the risk factors associated with hemoglobinuria after ultrasonography-guided percutaneous microwave ablation for large hepatic cavernous hemangiomas.

\section{RESULTS}

\section{Ablation efficacy}

All patients were performed MWA successfully. Technical effectiveness rate was $100 \%$ with a mean ablation time of $1251.2 \pm 535.1$ (range 480-2730) seconds. Within 3 days after ablation, 44 lesions were necrosis completely, and 5 lesions was more than $90 \%$ necrosis due to the peripheral of the tumor adjacent to dangerous location. The mean tumor volume shrinkage rate was $53.05 \pm 23.81 \%$ within 3 days after ablation. (Figure 1) Out of 20 patients who had hemoglobinuria after microwave ablation, the color of urine and urine routine test recovered normally gradually after basification of urine and hydration treatment in 19 cases. One case developed acute kidney injury (AKI) shortly after MWA, caused by massive heat-induced intravascular hemolysis. Lab results showed AKI (creatinine $227 \mathrm{micromol} / \mathrm{l}$ and urea $13.8 \mathrm{mmol} / 1$ in the second day after MWA, creatinine $353 \mathrm{micromol} / 1$ and urea $15.1 \mathrm{mmol} / 13$ days later after MWA). Because of progressive dyspnea and ongoing anuria haemodialysis through a femoral catheter was started. After 12 hemodialysis, 32 days later, the renal function gradually recovered and dialysis was stopped and the patient was discharged from the hospital 34 days after the procedure.

The preoperative characteristics and postoperative findings of the patients are shown in Table 1. Tumor maximum diameter was significantly larger among the patients with hemoglobinuria than among those with non-hemoglobinuria after MWA $(p=0.007)$. Ablation time was significantly longer among the patients with hemoglobinuria than among those with nonhemoglobinuria after MWA $(p=0.004)$. The number of antenna insertions was significantly more among the patients with hemoglobinuria than among those with nonhemoglobinuria after MWA $(p=0.005)$. Patients with hemoglobinuria use more energy than non hemoglobinuria patients after MWA $(p=0.001)$. There were no significant differences in liver function and renal function between the two groups before MWA. There were significant differences in ALT and AST level and renal function between the two groups in the second day after MWA $(p<0.05)$.

\section{Risk factors of hemoglobinuria}

By univariate analysis, tumor maximum diameter $(p=0.017)$, ablation time $(p=0.01)$, and the number of antenna insertions $(p=0.01)$ were statistically significant risk factors after MWA for HCHs. By multivariate analysis, the ablation time ( $p=0.021$; Odds Ratio, $1.005)$, and the number of antenna insertions ( $p=0.036$; Odds Ratio, 3.568) were the independent risk factors associated with hemoglobinuria after MWA for HCHs. (Table 2) When analyzed with use of ROC curves, ablation time (AUC, 0.759; 95\% CI: 0.626, 0.892; $P=0.002$ ) and the number of antenna insertions (AUC, $0.722 ; 95 \% \mathrm{CI}$ : $0.573,0.870 ; P=0.009$ ) were also significant factors for predicting hemoglobinuria. The cutoff value for ablation time in predicting the presence of hemoglobinuria was 1185s (sensitivity, 75\%; specificity, 69\%). The cutoff value for the number of antenna insertions in predicting the presence of hemoglobinuria was 4.5 (sensitivity, 55\%; specificity, 83\%) (Table 3).

\section{DISCUSSION}

For most patients with HCHs, open or laparoscopic surgical resection has been considered the preferred

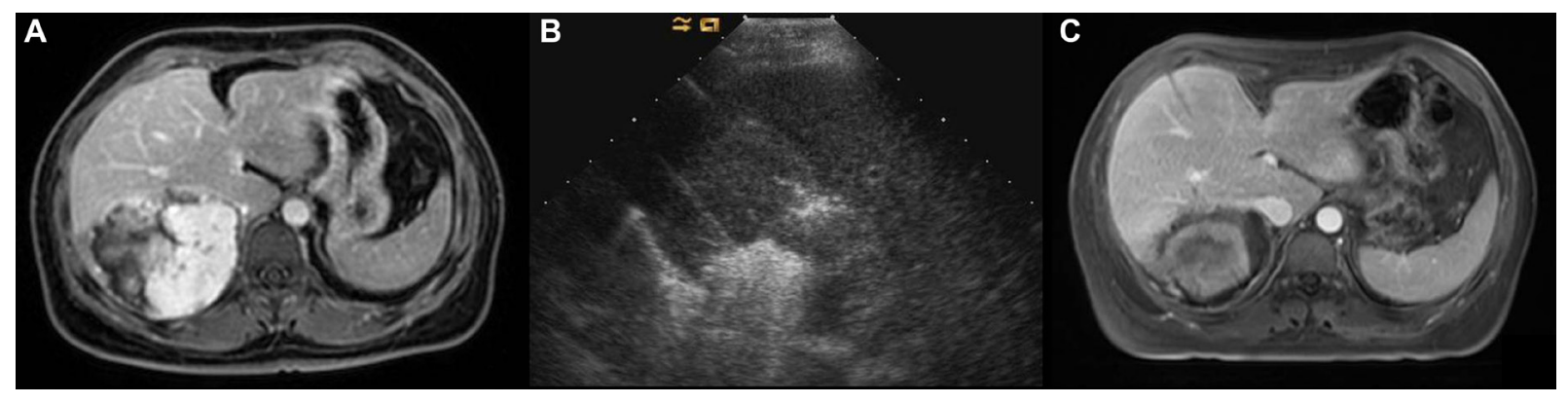

Figure 1: Images in a 39-year-old-woman who underwent MWA for hepatic cavernous hemangioma $10.4 \mathrm{~cm}$ in diameter. (A) Preoperative contrast-enhanced MRI showed the hemangioma was hyperenhanced in portal vein phase in right lobe. (B) US image showed the two antennae were placed into the tumor and ablated simultaneously. (C) Postoperative contrast-enhanced MRI showed complete necrosis of the tumor and and the maximum diameter was reduced to $7.8 \mathrm{~cm} 3$ days after MWA. 
Table 1: Comparison of clinicalparameters between hemoglobinuriagroup and non-hemoglobinuria group patients after microwave ablation for large hepatic cavernous hemangiomas

\begin{tabular}{|c|c|c|c|}
\hline Variable & hemoglobinuria $(n=20)$ & Non-hemoglobinuria $(n=29)$ & $P$ value \\
\hline Age (y) & $43.85 \pm 7.31$ & $42.75 \pm 8.89$ & 0.653 \\
\hline Gender (women/men) & $15 / 5$ & $21 / 8$ & 0.840 \\
\hline Max diameter $(\mathrm{cm})$ & $8.26 \pm 1.92$ & $6.89 \pm 1.48$ & 0.007 \\
\hline Ablation time (s) & $1506.00 \pm 440.86$ & $1075.51 \pm 529.82$ & 0.004 \\
\hline Tumor volume (ml) & $156.24 \pm 80.89$ & $119.62 \pm 71.45$ & 0.101 \\
\hline No. of antenna insertion & $4.80 \pm 1.32$ & $3.72 \pm 1.22$ & 0.005 \\
\hline Energy (J) & $154650 \pm 59004$ & $92151 \pm 36367$ & 0.001 \\
\hline ALT-pre (U/L) & $18.55 \pm 11.71$ & $16.65 \pm 12.02$ & 0.591 \\
\hline AST-pre (U/L) & $17.10 \pm 6.54$ & $15.75 \pm 4.53$ & 0.408 \\
\hline STB-pre (U/L) & $13.41 \pm 4.56$ & $11.75 \pm 3.97$ & 0.195 \\
\hline ALP-pre (U/L) & $60.77 \pm 14.26$ & $58.20 \pm 11.10$ & 0.495 \\
\hline Cre-pre $(\mu \mathrm{mol} / \mathrm{L})$ & $67.81 \pm 12.42$ & $63.60 \pm 11.56$ & 0.239 \\
\hline BUN-pre (mmol/L) & $4.70 \pm 1.25$ & $4.36 \pm 1.13$ & 0.329 \\
\hline ALT-post (U/L) & $216.42 \pm 189.54$ & $100.22 \pm 61.70$ & 0.003 \\
\hline AST-post (U/L) & $313.71 \pm 281.30$ & $145.73 \pm 116.43$ & 0.006 \\
\hline STB-post (U/L) & $41.07 \pm 31.86$ & $27.37 \pm 16.31$ & 0.054 \\
\hline ALP-post (U/L) & $56.52 \pm 12.99$ & $57.45 \pm 12.45$ & 0.823 \\
\hline Cre-post $(\mu \mathrm{mol} / \mathrm{L})$ & $90.08 \pm 47.12$ & $59.87 \pm 17.04$ & 0.004 \\
\hline BUN-post (mmol/L) & $6.95 \pm 6.04$ & $3.82 \pm 1.59$ & 0.013 \\
\hline Tumor reduction rate $(\%)$ & $52.14 \pm 25.35$ & $53.97 \pm 22.97$ & 0.832 \\
\hline
\end{tabular}

Abbreviations; ALT: alanine aminotransferase; AST: aspartate aminotransferase; STB: total bilirubin; ALP: alkaline phosphatase; Cre: creatinine; BUN: blood urea nitrogen; ${ }^{*}$-pre: index of preablation; ${ }^{*}$-post: index of postablation.

choice of treatment [17]. However, it is important to keep in mind that many of the trade-offs encountered during the treatment of cancer are not applicable to HCHs due to their benign natural history. Thus, a highly effective but morbid treatment would be a poor choice for most patients. Percutaneous thermal ablation, such as RFA and MWA, was safe, well-tolerated, and effective treatment method for most patients with HCHs [9-12]. However, due to the characteristics of the hemangioma itself, thermal ablation for hemangiomas may lead to a mass of red blood cells undergoing budding and fragmentation, which presumably resulted in a massive heat-induced intravascular hemolysis. Massive hemolysis can lead to various degrees of hemoglobinuria, hemolytic jaundice, anemia, and renal damage [11]. Determining risk factors associated with hemoglobinuria after thermal ablation for large $\mathrm{HCHs}$ is beneficial for reducing the risk of ablation, while there have been no report about it.

In our study, tumor maximum diameter, ablation time and the number of antenna insertions were statistically significant risk factors by univariate analysis. By multivariate analysis, we discovered that long ablation time and more antenna insertions during MWA can lead to hemoglobinuria with statistically significant cutoff values of 1185 s (nearly 20 mins) and 4.5, respectively. Therefore, when treating large $\mathrm{HCHs}$ with MWA, the number of antenna insertions and the ablation time should be reduced to the absolute minimum, in order to prevent hemoglobinuria. Van Tilborg AA et al. reported two patients with very large symptomatic $\mathrm{HCHs}$ who occurred hemoglobinuria and developed acute kidney injury shortly after bipolar RF ablation, and thought it was related with the size of the ablation zone and length of the procedure [14]. The goal of MWA for hemangioma and other benign lesions is tumor conformal necrosis or most part necrosis, so the ablation zone is basically the same as the tumor volume, but tumor volume were not statistically significant risk factors by univariate analysis, while the maximum tumor diameter is statistically significant only in univariate analysis, not in multivariate analysis in our study. During thermal ablation for $\mathrm{HCHs}$, hemoglobin is released upon erythrocyte destruction and is filtered by the glomerulus into the urinary space. In the urinary space, hemoglobin is degraded and releases hemepigments which are toxic to the kidney. Hemepigments can cause tubular injury by (1) tubular obstruction, (2) damage due to direct proximal tubular cell injury, and (3) vasoconstriction, resulting in reduced blood flow in the outer medulla [18]. So, proper 
Table 2: Univariate and multivariate analysis of clinical parameters related to hemoglobinuria after microwave ablation for large hepatic cavernous hemangiomas

\begin{tabular}{lcccc}
\hline \multirow{2}{*}{ Factor } & \multicolumn{2}{c}{ Univariate analysis } & \multicolumn{2}{c}{ Multivariate analysis } \\
\cline { 2 - 5 } & $\boldsymbol{P}$ Value & Odds Ratio & $\boldsymbol{P}$ Value & Odds Ratio \\
\hline Age & 0.645 & 1.017 & 0.872 & 0.987 \\
Gender & 0.840 & 1.143 & 0.093 & 27.720 \\
Max diameter & 0.017 & 1.668 & 0.860 & 1.104 \\
Tumor volume & 0.108 & 1.007 & 0.424 & 1.011 \\
ALT level & 0.584 & 1.014 & 0.155 & 1.171 \\
AST level & 0.408 & 1.048 & 0.906 & 0.970 \\
STB level & 0.197 & 1.100 & 0.320 & 1.196 \\
BUN level & 0.323 & 1.286 & 0.498 & 1.505 \\
Cre level & 0.236 & 1.031 & 0.126 & 1.126 \\
Ablation time & 0.010 & 1.002 & 0.021 & 1.005 \\
NO. of antenna insertion & 0.010 & 1.971 & 0.036 & 3.568 \\
\hline Abbrat
\end{tabular}

Abbreviations; ALT: alanine aminotransferase; AST: aspartate aminotransferase; STB: total bilirubin; ALP: alkaline phosphatase; Cre: creatinine; BUN: blood urea nitrogen.

Table 3: Sensitivity, specificity, and cutoff values for risk factors in predicting the presence of hemoglobinuria after microwave ablation for large hepatic cavernous hemangiomas

\begin{tabular}{llll}
\hline Factors & Cutoff value & Sensitivity (\%) & Specificity (\%) \\
\hline Ablation time & $1185 \mathrm{~s}$ & 75 & 69 \\
No. of antenna insertions & 4.5 & 55 & 83 \\
\hline
\end{tabular}

treatments including adequate hydration to decrease the $\mathrm{Hb}$ concentration in circulation system, alkalization of urine to make $\mathrm{Hb}$ crystal dissolution and prevent tubular obstruction and even acute kidney injury.

This study had some limitations. First, hemoglobinuria was qualitatively determined by urine routine without quantitative analysis. Second, the present study was a retrospective analysis which was performed in a single institution. The results must be confirmed in another cohort or in a prospective multicenter-study.

\section{MATERIALS AND METHODS}

\section{Patients}

From January 2011 to December 2016, 49 patients (36 females, 13 male; average age $43.20 \pm 8.22$ years) with 51 giant hepatic hemangiomas (mean maximum diameter $7.45 \pm 1.78 \mathrm{~cm}$, range $4.1-12.6 \mathrm{~cm}$ ) treated with image-guided percutaneous MWA were reviewed in this study. Among them, 20 patients had hemoglobinuria confirmed by urine routine after MWA. The patients were divided into two groups: hemoglobinuria group and nonhemoglobinuria group based on whether hemoglobinuria occurred after MWA.

Inclusion criteria for performing MWA were (1) definite diagnosis of a giant cavernous hemangioma $>4$ $\mathrm{cm}$ based on the typical enhancement pattern on contrastenhanced multiphase computed tomography (CT) or magnetic resonance imaging (MRI); (2) clinical symptoms typically caused by the giant hemangioma present for at least 1 year, including abdominal pain, nausea, vomiting, abdominal fullness. The diagnoses of $\mathrm{HCHs}$ were proven pathologically in all patients using US-guided core needle biopsy followed by ablation. This clinical application was approved by our institutional human research review committee. Written informed consent was obtained from all patients.

\section{Microwave equipment and ablation technique}

All treatments were performed in our institution and were carried out under US guidance with the patients under unconscious intravenous anesthesia (Propofol, 6-12 mg/kg/h; Ketamine, 1-2 mg/kg) in the operating room. The $2450 \mathrm{MHz}$ or $915 \mathrm{MHz} \mathrm{MW}$ system was used. The $2450 \mathrm{MHz}$ MW system (KY2000, Kangyou Medical, China) consists of three independent MW generators, three flexible coaxial cables and three water-pumping machines, which can drive three 15-gauge cooled-shaft antennae $(1.1 \mathrm{~cm}$ antenna tip) simultaneously. The $915 \mathrm{MHz} \mathrm{MW}$ system (KY-2001, Kangyou Medical, China) consists of two independent MW generators, two flexible coaxial cables 
and two water-pumping machines, which can drive two 15 -gauge cooled-shaft antennae $(2.2 \mathrm{~cm}$ antenna tip) simultaneously. The two MW system generators are capable of producing $1-100 \mathrm{~W}$ of power output. All therapy was performed by two experienced radiologists according to the preoperative planning. Hydrodissection technique and thermal monitoring technique were applied for hemangiomas abutting vital structures to avoid thermal damage. The detailed procedures of ultrasound-guided MWA in patients with liver tumors were described in our previous publications $[15,16]$.

\section{Evaluation methods}

The patients' blood tests (hepatic and renal function) and urine routine were taken before and after MWA. In the urine routine, dry chemistry analysis was used to detect $\mathrm{Hb}$ and flow cytometry was used to detect the red blood cells (RBCs). Hemoglobinuria was determined with the results of $\mathrm{Hb}$ positive and RBCs negative [13]. The wine-colored hemoglobinuria can be visually observed while deep-yellow-colored hemoglobinuria only can be detected by urine routine. Once abnormal results were discovered after MWA, hepatic or renal functions were tested every other day, and urine routine would be tested every time the patient urinated until all the results were normal. The data of tumor volume, ablation energy and ablation time in this study were all from one-session MWA. The tumor volume was calculated by the equation $\mathrm{V}=\pi^{*} \mathrm{a} * \mathrm{~b} * \mathrm{c} / 6$, where $\mathrm{a}$, $b$ and $c$ were three dimensions of the tumor measured by contrast-enhanced ultrasound. The number of antenna insertions was defined as the total number of antenna placements for each patient during ablation.

\section{Statistical analysis}

Data analysis was performed using SPSS17.0 for windows (SPSS Inc, Chicago, IL, USA) and the continuous data were expressed as means \pm standard deviations (SD). Data were analyzed between the hemoglobinuria group and non-hemoglobinuria group by using the Student $t$ test for unpaired data and the $\mathrm{x}^{2}$ test and Fisher exact test as appropriate. 11 related risk factors, including gender, age, tumor maximum diameter, tumor volume, ablation time, the number of antenna insertions, alanine aminotransferase (ALT), aspartate aminotransferase (AST), total bilirubin (STB), blood urea nitrogen (BUN) and creatinine (Crea) were analyzed using univariate and multivariate binary logistic regression model method. Receiver operating characteristic (ROC) curves were constructed, and the area under the ROC curve (AUC) was calculated by using the trapezoidal rule. Optimal cutoff values for risk factors were selected to maximize sensitivity, specificity. All of the statistical tests were two-sided, and significance was set at $p<0.05$.

\section{CONCLUSIONS}

In conclusion, the ablation time and No. of antenna insertion were the independent risk factors associated with hemoglobinuria when MWA for large HCHs. Less than 5 of antenna insertions and less than 20 mins of ablation time may therefore be recommended in patients with MWA of large $\mathrm{HCHs}$, in order to reduce the occurrence of hemoglobinuria.

\section{Abbreviations}

hepatic cavernous hemangiomas, $\mathrm{HCHs}$; microwave ablation, MWA; receiver operating characteristic, ROC; vascular endothelial growth factor, VEGF; radiofrequency ablation, RFA; acute kidney injury, AKI.

\section{Author contributions}

The authors alone are responsible for the content and writing of the paper.

\section{CONFLICTS OF INTEREST}

The authors report no conflicts of interest.

\section{FUNDING}

This study was supported by the National Scientific Foundation Committee of China (grant numbers 81201167) and Beijing Nova Program (xx2013108).

\section{REFERENCES}

1. Wanless IR. Micronodular transformation (nodular regenerative hyperplasia) of the liver: a report of 64 cases among 2,500 autopsies and a new classification of benign hepatocellular nodules. Hepatology. 1990; 11:787-97.

2. Schnelldorfer T, Ware AL, Smoot R, Schleck CD, Harmsen WS, Nagorney DM. Management of giant hemangioma of the liver: resection versus observation. J Am Coll Surg. 2010; 211:724-730.

3. Corigliano N, Mercantini P, Amodio PM, Balducci G, Caterino S, Ramacciato G, Ziparo V. Hemoperitoneum from a spontaneous rupture of a giant hemangioma of the liver: report of a case. Surg Today. 2003; 33:459-463.

4. Watzke HH, Linkesch W, Hay U. Giant hemangioma of the liver (Kasabach-Merritt syndrome): successful suppression of intravascular coagulation permitting surgical removal. J Clin Gastroenterol. 1989; 11:347-350.

5. Singh RK, Kapoor S, Sahni P, Chattopadhyay TK. Giant haemangioma of the liver: is enucleation better than resection? Ann R Coll Surg Engl. 2007; 89:490-3. 
6. Giavroglou C, Economou H, Ioannidis I. Arterial embolization of giant hepatic hemangiomas. Cardiovasc Intervent Radiol. 2003; 26:92-6.

7. Gaspar L, Mascarenhas F, da Costa MS, Dias JS, Afonso JG, Silvestre ME. Radiation therapy in the unresectable cavernous hemangioma of the liver. Radiother Oncol. 1993; 29:45-50.

8. Mahajan D, Miller C, Hirose K, McCullough A, Yerian L. Incidental reduction in the size of liver hemangioma following use of VEGF inhibitor bevacizumab. J Hepatol. 2008; 49:867-80.

9. Ziemlewicz TJ, Wells SA, Lubner MA, Musat AI, Hinshaw JL, Cohn AR, Lee FT Jr. Microwave ablation of giant hepatic cavernous hemangiomas. Cardiovasc Intervent Radiol. 2014; 37:1299-305.

10. Park SY, Tak WY, Jung MK, Jeon SW, Cho CM, Kweon YO, Kim KC. Symptomatic-enlarging hepatic hemangiomas are effectively treated by percutaneous ultrasonography-guided radiofrequency ablation. J Hepatol. 2011; 54:559-565.

11. Gao J, Ke S, Ding XM, Zhou YM, Qian XJ, Sun WB. Radiofrequency ablation for large hepatic hemangiomas: Initial experience and lessons. Surgery. 2013; 153:78-85.

12. Sharpe EE 3rd, Dodd GD 3rd. Percutaneous radiofrequency ablation of symptomatic giant hepatic cavernous hemangiomas: report of two cases and review of literature. J Vasc Interv Radiol. 2012; 23:971-975.

13. Veerreddy P. Hemoglobinuria misidentified as hematuria: review of discolored urine and paroxysmal nocturnal hemoglobinuria. Clin Med Insights Blood Disord. 2013; 6:7-17.
14. van Tilborg AA, Dresselaars HF, Scheffer HJ, Nielsen K, Sietses C, van den Tol PM, Meijerink MR. RF Ablation of Giant Hemangiomas Inducing Acute Renal Failure: A Report of Two Cases. Cardiovasc Intervent Radiol. 2016; 39:1644-1648.

15. Liu F, Yu X, Cheng Z, Han Z, Sun Y, Liang P, Zhou F. Comparison of ultrasonography-guided percutaneous microwave ablation for subcapsular and nonsubcapsular hepatocellular carcinoma. Eur J Radiol. 2017; 91:93-98.

16. Yu J, Yu XL, Han ZY, Cheng ZG, Liu FY, Zhai HY, Mu MJ, Liu YM, Liang P. Percutaneous cooled-probe microwave versus radiofrequency ablation in early-stage hepatocellular carcinoma: a phase III randomised controlled trial. Gut. 2017; 66:1172-1173.

17. Miura JT, Amini A, Schmocker R, Nichols S, Sukato D, Winslow ER, Spolverato G, Ejaz A, Squires MH, Kooby DA, Maithel SK, Li A, Wu MC, et al. Surgical management of hepatic hemangiomas: a multi-institutional experience. HPB (Oxford). 2014; 16:924-928.

18. Heyman SN, Rosen S, Fuchs S, Epstein FH, Brezis M. Myoglobinuric acute renal failure in the rat: a role for medullary hypoperfusion, hypoxia, and tubular obstruction. J Am Soc Nephrol. 1996; 7:1066. 